\title{
Quantification of Grain Boundary Segregation Monolayers by X-ray Spectroscopy in a Scanning Electron Microscope
}

\author{
P. Nowakowski*, F. Christien*, M. Allart*, Y. Borjon-Piron*, R. Le Gall*, J.C. Ménard**, H. \\ Mantz*** \\ * Laboratoire Génie des Matériaux et Procédés Associés (LGMPA), Université de Nantes, \\ Polytech'Nantes, Rue Christian Pauc, BP 50609, 44306 Nantes Cedex 3, France \\ ** Carl Zeiss NTS sas, 27 rue des peupliers, 92752 Nanterre Cedex, France \\ *** Carl Zeiss NTS GmbH, Research \& Development, Carl-Zeiss-Straße 56, D-73447 \\ Oberkochen, Germany
}

Segregation of impurity elements such as sulphur to grain boundaries of metals leads to dramatic degradation of mechanical properties. Extensive effort has been made for decades to detect and to quantify monolayers of solute segregated at grain boundaries. The most widespread techniques for grain boundary chemistry have been Auger Electron Spectroscopy and, more recently, Transmission Electron Spectroscopy associated with X-ray or Electron Energy Loss spectroscopies.

In this work, we have developed a new technique for the quantification of grain boundary segregation monolayers by Wavelength Dispersive X-ray Spectroscopy (WDS) in a high current Scanning Electron Microscope (SEM) [1,2].

The material studied here is pure nickel containing $5 \mathrm{wt}$. ppm of sulphur in solid solution. Heat treatments have been carried out on the material to induce sulphur grain boundary segregation. The samples to be analyzed are fractured at $77 \mathrm{~K}$ using a conventional tensile machine and then introduced in the SEM chamber. A grain boundary fracture is usually obtained as shown in Fig. 1a. The fracture surface is analyzed in the new MERLIN FEG-SEM from Carl ZEISS NTS fitted with an IncaWave WDS spectrometer from Oxford Instruments. For each sample, about 20 grain boundary facets are analyzed at an accelerating voltage of $20 \mathrm{kV}$ and a beam current of about 400 nA (Fig. 1b).

The sulphur K $\alpha$ line is clearly detected on the WDS spectra. Although the volume of analysis is "large" in nickel at $20 \mathrm{kV}$, there is no contribution of the bulk to the sulphur peak since the bulk sulphur content is negligible $(\sim 5 \mathrm{ppm})$. The sulphur $\mathrm{K} \alpha$ line observed on the WDS spectra can be attributed to a fraction of monolayer on the fracture surface. The sulphur grain boundary concentration $\mu$ can be expressed as:

$$
\mu=K \frac{I}{I_{S t d}} \cos \theta
$$

where $K$ is matrix correction factor $\left(256.1 \mathrm{~g} . \mathrm{cm}^{-2}\right), I$ and $I_{S t d}$ are the sulphur $\mathrm{K} \alpha$ peak height measured on the sample and on a standard material respectively, $\theta$ is the tilt angle. We have developed an original method to determine the tilt angle of the analyzed surface from the absorbed specimen current:

$$
\cos \theta=\frac{0.36}{1-C_{A B S} / C_{B}}
$$

where $C_{B}$ is the beam current ( $\left.400 \mathrm{nA}\right)$ ant $C_{A B S}$ is the specimen absorbed current.

Fig. 2a shows the distribution of the sulphur grain boundary concentration over the analyzed facets for 2 different heat treatments. Fig. $2 b$ presents the kinetics of sulphur grain boundary segregation at $750^{\circ} \mathrm{C}$ measured by WDS. 


\section{Conclusion}

1. WDS can be used to quantify monolayer grain boundary segregation in a high current FEGSEM.

2. The WDS technique is insensitive to surface contamination and can be used on ex-situ fractured samples (contrary to Auger spectroscopy).

3. The precision and limit of detection of WDS is about $2 \mathrm{ng} \cdot \mathrm{cm}^{-2}$ (or $2 \%$ of a monolayer).

\section{References}

[1] F. Christien, R. Le Gall, Surface Science 602 (2008) 2463.

[2] P. Nowakowski, F. Christien, M. Allart, Y. Borjon-Piron, R. Le Gall, Surface Science (2011), doi:10.1016/j.susc.2011.01.032
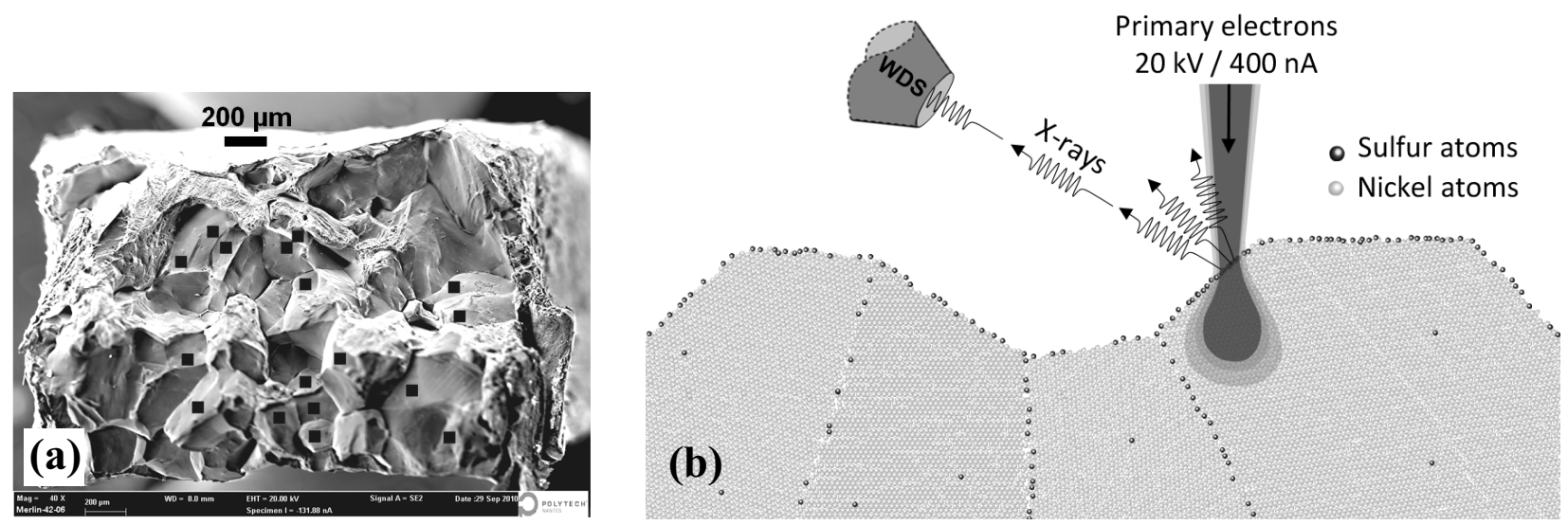

FIG. 1. (a) SEM observation of a typical grain boundary fracture of nickel due to sulphur segregation. (b) Principle of the technique: a high current electron beam ( 400 nA) is focused on a grain boundary facet; the emerging sulphur X-ray intensity is then analysed using WDS.

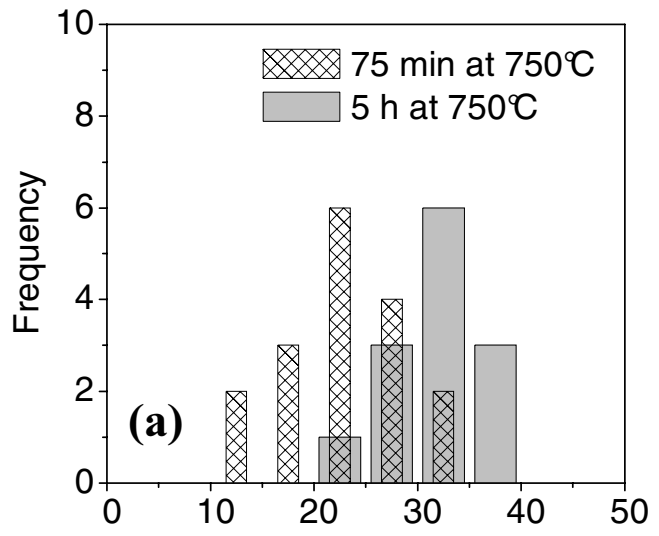

Sulphur grain boundary concentration $\left(\mathrm{ng} . \mathrm{cm}^{-2}\right)$

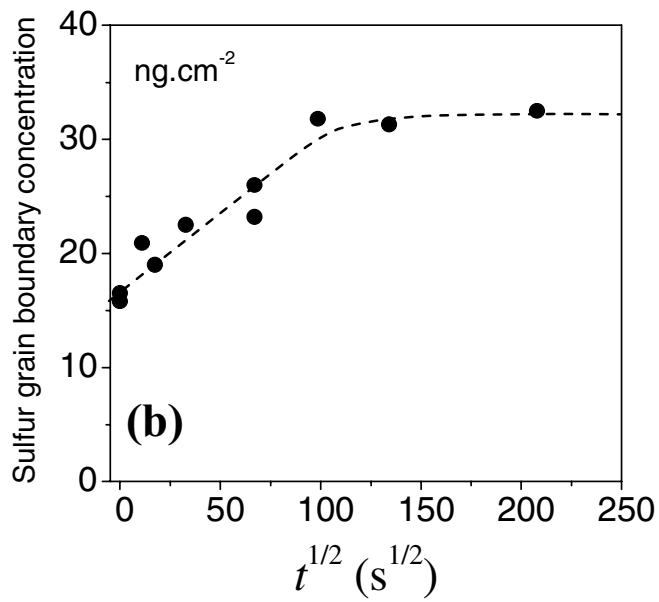

FIG. 2. (a) Distribution of the WDS quantification over the analyzed facets for two different heat treatments. (b) Kinetics of sulphur grain boundary segregation at $750^{\circ} \mathrm{C}$ measured by WDS. 